\title{
EL CURRICULUM DE CIENCIAS EN GRAN BRETAÑA
}

\author{
PINTÓ, R.
}

Escola de Magisteri de Sant Cugat

Universitat Autónoma de Barcelona

\section{INTRODUCCION}

En el I Simposium Internacional de Didáctica General y Didácticas especiales celebrado el pasado octubre en Murcia tuvimos el honor de contar con la asistencia del profesor Paul J. Black director del Centre for Science and Mathematics Education del Chelsea College de Londres.

Una de sus conferencias consistió en una visión global del panorama actual de la Enseñanza de las Ciencias en Gran Bretaña. A lo largo de este articulo pretendemos glosar un aspecto particularmente interesante de dicha conferencia: el tema del curriculum de Ciencias.

P.J. Black planteó los debates que en su pais se están produciendo acerca de qué hay que enseñar de Ciencias en la Escuela Primaria y Secundaria.

\section{ESCUELA PRIMARIA}

En la Escuela primaria que en Gran Bretaña llega hasta los 11 años. la enseñanza de las Ciencias es muy heterogénea. Dice Black que muchos profesores abordan con poca frecuencia los temas de Ciencias en sus clases porque se sienten inseguros para ello. Otros debido a la falta de una buena preparación tienden a enseñar algo de Ciencias como un cuerpo de conocimientos verdaderos y acabados.

En la última década se han realizado nuevos proyectos que pretendían presentar una alternativa a esta situación. Tales son el Nuffield Junior Science Project, el Science 5/13 y recientemente el Learning through Science. Todos ellos han producido nuevos materiales para el currículum y guias para el profesor centrándose únicamente en los procesos de la Ciencia que los niños deben adquirir para un enfoque científico de los sucesos de la vida cotidiana.

La cuestión que se plantea, se debate insistentemente y sigue sin una solución definitiva es: ¿Son importantes los contenidos científicos sobre los que versar la Enseñanza Primaria? Y por ello ies necesario elaborar un programa común para todas las escuelas?

Para algunos puede incluso parecer sorprendente la pregunta. Si consideran que las Ciencias en la Escuela primaria son únicamente unos contenidos entonces la elección de dichos contenidos será fundamental. Pero en los últimos 15 años se ha sostenido con frecuencia que para las Ciencias en la Escuela elemental no tiene relevancia la elección de contenidos que se haga, que lo importante es experimentar, tener habilidad por cuestionar y fomentar unas actitudes científicas. De acuerdo con este punto de vista, lo esencial es la manera con que se aborda un tema o una cuestión y no el conocimiento al que conduce.

W. Harlen del Centre for Science Education, Chelsea College, resume en cinco puntos los argumentos de los que sostienen esta postura que implica no elaborar un programa de contenidos común para todas las escuelas:

1. "Si decimos que queremos conseguir que los niños se formulen cuestiones y descubran por sí mismos la respuesta esto implica llevar a cabo investigaciones hasta que se llega a una conclusión $y$ son dificiles de realizarlas plenamente si un programa extemo fija las cuestiones a plantearse e indica los caminos o las cosas sobre las que hay que tener curiosidad.

2. Por otro lado no podemos saber lo que es mejor enseñar porque lo que es importante hoy puede no serlo mañana o ni tan siquiera ser cierto. Por tanto lo que conviene es equipar a los niños para que sepan aprender y descubrir por sí mismos; esto hará que en su dia consigan dominar la variedad de conocimientos que deseen.

3. Los programas centralizados no pueden tener en cuenta la diversidad en medios locales o re- 
gionales con to que se hace dificil un enraizamiento con el medio que rodea al niño.

4. Si las actividades científicas están determinadas por un programa que se sigue, será dificil integrarlas con el trabajo en otras áreas del curriculum escolar, no será posible la interdisciplinariedad.

5. Para que reaimente los niños se sientan motiva. dos para aprender unos hábitos y para desarrollar unos conceptos es necesario que elijan el tema de estudio de acuerdo con sus intereses. Muchas de las actividades de un programa prescrito resultarán sin sentido hasta que los niños puedan conectarlas con sus ideas."

Algunos de estos argumentos tienen fácil réplica dice Harlen. Por ejemplo, en la práctica es muy difícil seguir el interés de todos los niños de una clase. Y este enfoque contrario a la implantación de un programa común tiene otros inconvenientes. Supone un estilo de maestro con gran flexibilidad, capaz de partir del punto en que cada niño se encuentra, con gran seguridad en cómo plantear ciertas cuestiones y en cubrir en cualquier caso el núcleo central de cualquier programa o guía básicos a partir de los intereses que los niños manifiestan.

Pero ¿qué ocurre cuando los niños se encuentran con maestros sin tal cantidad de requisitos? ¿Cómo asegurar que todos los niños tendrán oportunidad de recibir las ideas básicas que sean los cimientos para la comprensión gradual de su mundo?

Harlen plantea estos interrogantes y a la vez expone las tesis de los defensores de un programa de contenidos común:

1. «Si como educadores tenemos la obligación de alfabetizar a nuestros alumnos, hay ideas y conceptos básicos que inexorablemente debemos proporcionarles para ayudarles a comprender el mundo que les rodea.

2. La introducción de conceptos debe ser graduada y ordenada. No se puede enseñar cualquier cosa si aún no se conocen las ideas elementales, previas. Es necesario poner bien las ideas básicas escogidas de modo que contribuyan a una futura comprensión.

3. Seguir un programa no implica necesariamente dar menor inportancia al desartollo de la capacidad de investigar y a la adquisición de clertas actitudes. Es incluso más fácil porque los problemas planteados están controlados y los materiales estructurados. Esto hace posible resul. tados más satisfactorios ya que los niños no se pierden ante la multitud de variables incontrola- bles que conlleva el estudio de un problema real cualquiera sin que las actividades hayan sido cuidadosamente probadas.

4. Por otro lado son los maestros quienes saben cuáles son los conocimientos que es más probable que utilicen los niños, ellos son pues los que deben llevar la batuta. Ante la gran variedad de cosas de su alrededor ¿qué tienen que escoger los niños?

5. Y, finalmente, se citan con frecuencia algunas razones de naturaleza más práctica. Por ejemplo: ha de empezarse en la Escuela Primaria a proporcionar algunos contenidos porque no hay tiempo para llegar a todo en la Secundaria. O bien: Los maestros no han recibido una formación suficiente como para que puedan elaborarse unos programas de contenidos que sean equilibrados, hay pues que suministrárselos."

También se puede en este caso poner objeciones a algunos de los argumentos. Quizás la mayor sea el de la arrogancia implícita de que conocemos el mejor contenido. $Y$ es dificil estar seguros de cuá les son las ideas o conceptos que proporcionan una base firme sobre la que asentar más tarde las nociones más sofisticadas. $\mathrm{E}$ incluso, si los supiéramos, sería difícil que concordaran con los criterios de ser interesantes para los niños o apropiados para comprender el entorno inmediato.

Por otro lado, el contenido común para todos los niños generalmente significa que hacen todos las mismas actividades sin tener en cuenta no sólo sus intereses sino también sus capacidades. Un programa individualizado no es necesariamente la solución ya que el tiempo dedicado a la realización de una tarea es solamente una variable que afecta al aprendizaje; la presentación del problema, los materiales utilizados, la cantidad y tipo de ayuda son también importantes. Puede ocurrir que sea necesaria una diversidad de contenidos para asimilar niños distintos las mismas ideas; entonces se han perdido las ventajas de un programa común.

Se hace pues difícil dar una respuesta estricta a la cuestión de si es o no necesaria la existencia de un programa para la Enseñanza de las Ciencias común a todas las Escuelas Primarias.

Lo que parece necesario es disponer de unas guías que sean suficientemente firmes para asegurar que el niño encuentra el abanico de ideas y fenómenos que parecen relevantes para la comprensión de su medio y a la vez suficientemente vagas para que los profesores puedan usar una variedad de caminos para llegar a ellos. 
Con el planteamiento de este debate de fondo, Black finalizó su disertación sobre el currículum de Ciencias en la Escuela primaria inglesa.

\section{ESCUELA SECUNDARIA}

En Gran Bretaña, la mayoría de los niños a los Il años pasan a la Escuela secundaria que comprende dos etapas: la Junior Secondary (11 a 13 años) y la Senior Secondary (13 a 16 años).

Durante los dos años de la Junior Secondary todos los niños hacen cursos en forma de Ciencia integrada o combinada.

También en este nivel hay discusión sobre el enfoque que hay que dar a la enseñanza, algunos creen que los cursos basados en los conceptos de la Ciencia, son demasiado avanzados dado el desarrollo cognitivo de los niños de estas edades.

Ha habido un cambio hacia cursos organizados entorno de temas $o$ aspectos que son probablemente de interés para los alumnos. Pero subsiste el problema de si hay que proporcionar y de qué manera algunos conceptos abstractos básicos y a la vez desarrollar las habilidades propias de los métodos de trabajo del científico.

La decisión gubernamental es la de organizar el curriculum cuidadosamente con el fin de compaginar las tres facetas: a partir del estudio de temas de la vida cotidiana introducir los conceptos bási$\cos$ y hacerlo mediante una terminologia que permita el desartollo de las habilidades del cientifico. Pero, aunque se han elaborado algunas propuestas, este curriculum es difícil de realizar.

Pero son los cursos del final de la escolaridad obligatoria, de los 13 a 16 años (Senior Secondary) los que han provocado un debate más intenso.

Se plantean fundamentalmente tres cuestiones:

1. ¿Las asignaturas de Ciencias han de ser optativas o no?

2. ¿Qué tipo de Ciencias hay que enseñar?

3. ¿La enseñanza debe hacerse con grupos de alumnos homogéneos o heterogéneos?

Veamos brevemente cómo se abordan estas cuestiones.

\subsection{La optatividad}

En Gran Bretaña a los 13 años los alumnos acostumbran a elegir entre las asignaturas de Ciencias.

A la hora de buscar trabajo se valora más a los que han pasado los exámenes de las tres Ciencias:
Física, Química y Biología por separado. Pero aunque sea mayor el prestigio de los que han seguido este tipo de exámenes, son pocos los alumnos que escogen las tres materias, esto les supondría $1 / 3$ del horario escolar. Eligen una o dos asignaturas $e$ incluso hay algunos (15\% a los $15-16$ años) que optan por no hacer ninguna asignatura de Ciencias por separado o bien por apuntarse a un curso de Ciencias generales que goza de un menor estatus.

Y este sistema de elección es muy polémico porque tiene efectos demasiado importantes. Por ejemplo, no haber escogido estudiar Física como asignatura por separado entre los 13 a 16 años supondrá no poder seguir estudios universitarios de Ciencias Físicas o de Ingenieria si posteriormente se desea.

Por esta razón, recientemente el Departamento de Educación del Gobiemo británico ha hecho la propuesta de disminuir en sus $2 / 3$ la cantidad de contenidos de los programas cortespondientes a los exámenes de certificado de las tres Ciencias. Esto hace posible que se impartan las tres materias en el mismo tiempo que antes se hacian dos.

\subsection{Orientación de los programas}

El punto de debate más intenso es qué tipo de Ciencia hay que enseñar al estudiante medio. Dice $P$. Black que aún muchos de los cursos están basados en el enfoque proporcionado hace tiempo por los mejores especialistas en cada materia. $Y$ así, los intentos de adaptación de estos enfoques para los alumnos menos capacitados no resultan ser más que una dieta diluida, de pobre calidad en la que los conceptos más abstractos se aprenden de memoria y en la que no se consigue interesar al alumno. Estos alumnos calificados de menos capaces son los que se estimula su inteligencia a través del trabajo práctico más que a través de abstracciones. Por esta razón, si la escolaridad obligatoria - abarca hasta los 16 años habrá que ofrecer una enseñanza para todos los alumnos que tenga como eje fundamental las necesidades del ciudadano medio. Esto que, desde la Association for Science Education, se está analizando a fondo para proponer una alternativa definida, parece que llevará a unos cursos con más énfasis en la tecnología y sus aplicaciones y con más atención a la historia y las implicaciones sociales de la Ciencia.

Se han elaborado programas de Ciencia integrada para estos niveles pero han tenido poca aceptación por parte de los profesores y de los que se dedican 
a la selección de personal en las empresas. Estos ven con mejores ojos los curricula vitae con las calificaciones tradicionales de tres asignaturas separadas y sospechan que los cursos de Ciencia integrada son más pobres en contenido y rigor. $\mathrm{Mu}$ chos profesores tampoco aceptan programas de esta índole o bien porque consideran que su profesionalidad depende del conocimiento que tengan de cada asignatura o bien porque encuentran que existen diferencias profundas en las finalidades $y$ en los métodos de las diversas Ciencias.

Pero si hay que evitar los efectos de una elección demasiado comprometedora a los 13 años y ofrecer una Ciencia útil al ciudadano medio, alguna fórmula de compromiso habrá que establecerse. Es con esta finalidad que recientemente se ha elaborado el proyecto Nuffield 13-16.

El Nuffield 13-16 consta de 44 módulos (libro del alumno y guía del profesor) con cierto encadenamiento entre unos y otros. El profesor puede escoger entre las diversas unidades y elaborar así su curso. Existen módulos básicos que como su nombre indica son los prerrequisitos para los posteriores y existen módulos Simples, módulos de Doble extensión y módulos $\mathrm{X}$ para la profundización en algún aspecto concreto. De la lectura de sus titulos podemos darnos cuenta que algunas unidades están basadas en conceptos mientras que otras lo están en temas de la vida cotidiana.

Los profesores pueden utilizar el material de varias maneras de acuerdo con las características del grupo clase. Hay actividades de diversa dificultad para adaptarse a un amplio abanico de capacidades pero el nivel de lenguaje utilizado es para ei alumno medio.

Buen número de profesores seleccionan unos módulos para hacer un curso de Ciencias generales para el primer curso de la Senior Secondary en el que acostumbran a haber grupos de alumnos heterogéneos y luego construyen cursos de Ciencias separadas que se apoyan en el anterior para los dos próximos años (es frecuente hacer grupos de alumnos homogéneos para los de 14 a 16 años).

A partir de esta síntesis de la disertación de J.P. Black nos podemos dar cuenta de la problemática del diseño del currículum de Ciencias para los diversos niveles en Gran Bretaña, problemática que no nos resulta ajena sino que está siendo motivo de debate también entre los enseñantes de este país referido a nuestras circunstancias. 